\title{
Arsenite suppresses angiogenesis of vascular endothelial cells mediated by Platelet
}

\section{Derived Growth Factor Receptor-beta}

Xiaotong Wang ${ }^{1 \#}$, Yan Mou ${ }^{1,3}$, Zhen Yue ${ }^{1}$, Haiying Zhang ${ }^{1}$, Xuejin $\mathrm{Su}^{1}$, Yang Wang ${ }^{1}$, Ronggui $\mathrm{Li}^{1} *$ and $\mathrm{Xin} \mathrm{Sun}^{2} *$

${ }^{1}$ Key Laboratory of Pathobiology, Ministry of Education, Norman Bethune College of Medicine, Jilin University, Changchun, China

${ }^{2}$ Life Science Research Center, Beihua University, Jilin, P.R. China

${ }^{3}$ The Second Hospital of Jilin University, Changchun, P.R. China

*Correspondence to: Dr. Ronggui Li, The Key Laboratory of Pathobiology, Ministry of Education, Norman Bethune College of Medicine, Jilin University, Changchun, 130021, P.R. China. Tel.: 86-431 85619481; Fax: 86-431-85619469; E-mail: lirg@jlu.edu.cn and Dr. Xin Sun, Life Science Research Center, Beihua University, Jilin, 132013, P.R. China. Tel.: 86-432-64608351; E-mail: sunxinbh@ 126.com.

${ }^{\#}$ These authors contributed equally to this work

\section{RUNNING TITLE}

Arsenite suppresses angiogenesis of HUVECs 


\section{ABSTRACT}

The present study aimed to investigate the effects of sodium arsenite $\left(\mathrm{NaAsO}_{2}\right)$ on the angiogenesis of human umbilical vein endothelial cells (HUVECs) and the mechanism involved. Firstly, a Matrigel-based in vitro angiogenesis assay demonstrated that arsenite suppressed the angiogenesis of HUVECs in a dose-dependent manner. Then by using a global inhibitor for multiple growth factor receptors (E7080) and a specific inhibitor of PDGFR-beta (CP-673451), we found that E7080 completely prevented and CP-673451 significantly decreased the angiogenesis of HUVECs. This suggested that angiogenesis of HUVECs depends on the signal pathway mediated by tyrosine kinase receptors and that among them, PDGFR-beta has an important regulatory function. Finally by using porcine aortic endothelial cells which stably express human PDGFR-beta, we found that arsenite suppressed the angiogenesis mediated by PDGFR-beta. Based on these results, we conclude that arsenite suppressed the angiogenesis of the vascular endothelial cells, that this effect is mediated by PDGFR-beta, and postulate that it might contribute to the injuries of blood vessel in arsenism.

KEY WORDS: Arsenic; Angiogenesis; Human Umbilical Vein Endothelial Cells; Platelet Derived Growth Factor Receptor 


\section{INTRODUCTION}

High arsenic levels of drinking water in endemic regions remain a major public health concern which affects more than a hundred million people worldwide (Rahman et al., 2009). Chronic exposure to inorganic arsenic has been shown to elicit multiple health disorders, such as skin lesions, cancers of the skin, lung, kidney, bladder, and liver, cardiovascular disease (Chang et al., 2004; Wu et al., 1989) and diabetes mellitus (Liu et al., 2015). The best known adverse effect of long-term arsenic exposure is gangrene, secondary to peripheral vascular disease (Tseng et al., 2007).

Healthy vascular endothelial cells are responsible for the maintenance of vascular integrity, repair of the injured vessel wall, and the neovascularization of damaged tissue (Mannarino and Pirro, 2008). Using human umbilical vein endothelial cells (HUVECs) as an in vitro experimental model it has been found that arsenic inhibits the proliferation of HUVECs by preventing cell cycle progression (Woo et al., 2005). Sodium arsenite enhanced the apoptosis of HUVECs by its effects on mitochondrial function (Shi et al., 2010). Lower concentrations (up to $1 \mu \mathrm{M}$ ) of sodium arsenite increase vascular tube formation (Kao et al., 2003). However, studies about the effects of arsenite on the angiogenesis of HUVECs have not been reported, nor have potential mechanisms been elucidated.

Angiogenesis is a major function of vascular endothelial cells (Hofer and Schweighofer, 2007), and, in tumor angiogenesis is largely mediated by alterations of receptor tyrosine kinase pathways (Zhang and Simons, 2014). They are a diverse group of transmembrane 
proteins which function by transducing growth factor signals from the external milieu to intracellular processes (Haluska and Adjei, 2001). A multi-targeted tyrosine kinase inhibitor lenvatinib (E7080) has been demonstrated to have anti-angiogenesis activities in colorectal cancer xenografts (Wiegering et al., 2014), in preclinical human thyroid cancer models (Zhang and Simons, 2014) and in patients with advanced solid tumors (Roberts et al., 2005). A selective inhibitor of PDGFR-beta kinase, CP-673,451, has also been shown to be capable of inhibiting PDGF-BB-stimulated angiogenesis in malignant tumors (Roberts et al., 2005). In the present study, we analyzed the effects of arsenite on the angiogenesis of HUVECs and the possible signals involved in order to explore the mechanisms through which arsenic causes injuries of blood vessels. 


\section{MATERIALS AND METHODS}

\section{Materials}

Human Umbilical Vein Endothelial Cells (HUVECs, Cat. No. 8000) and endothelial cell medium (ECM, Cat. No. 1001) were purchased from the ScienCell Research Laboratories (San Diego, USA). Porcine aortic endothelial cells with stably transfected human PDGF beta-receptors were from Professor Rainer Heuchel, Karolinska Institute, Sweden. IMDM (Cat. No. 21056023) was purchased from Gibco BRL (Rockville, USA). Fetal bovine serum (FBS, Cat. No. SH30071.03) was purchased from HyClone Inc. (Logan, USA). Endothelial cell growth supplement (ECGS, Cat. No. 1052) was purchased from ScienCell Research Laboratories (San Diego, USA). In Vitro Angiogenesis Assay Kit (Cat. No. ECM625) was purchased from Millipore (Billerica, USA). Calcein-AM (Cat. No. sc-203865) was purchased from Santa Cruz Biotechnology, Inc. (Dallas, USA). Sodium arsenite $\left(\mathrm{NaAsO}_{2}, \mathrm{CAS}\right.$ No. 7784-46-5) was purchased from Sigma-Aldrich (St. Louis, USA). E7080 (Cat. No. S1164) and CP-673451 (Cat. No. S1536) were purchased from Selleck Chemical (Huston, USA).

\section{Cell culture and treatments}

The HUVECs were grown in ECM medium containing $5 \%$ FBS and $1 \%$ endothelial cell growth supplement (ECGS). PDGFR-beta/PAE cells were grown in IMDM containing $10 \%$ FBS. Both cell types were incubated at $37^{\circ} \mathrm{C}$ in $5 \% \mathrm{CO}_{2}$ and a humidified atmosphere. HUVECs were used for all experiments at passages 2 to 6 . For arsenic treatment, the cells were plated in dishes of a $6 \mathrm{~cm}$ diameter at a density of $0.5 \times 10^{5} \mathrm{cells}$ per dish. After incubating them for 24 hours, the medium was exchanged with fresh medium containing 
various concentrations of arsenite or vehicle and incubated for different time, as indicated in the figures 1 to 5 .

\section{In vitro angiogenesis assay}

The angiogenesis of the cells was evaluated by a Matrigel in vitro angiogenesis assay technique. The assay was performed with a detailed procedure as described previously (Mou et al., 2016). Briefly, $100 \mu$ stock solution of Matrigel was added to each well in 48 -well plates and kept at $37^{\circ} \mathrm{C}$ for $30 \mathrm{~min}$ in order to form the Matrigel. Cell suspensions containing $3 \times 10^{4}$ cells in $100 \mu$ of ECM were seeded on the Matrigel of each well, and incubated for 6 hours. Then Calcein-AM $(0.1 \mathrm{mM})$ was directly added to each well for $20 \mathrm{~min}$ at $37^{\circ} \mathrm{C}$ to stain the cells which were imaged under a phase contrast microscope with an excitation wavelength of $490 \mathrm{~nm}$ and an emission wavelength of $515 \mathrm{~nm}$. For quantification, the values for the pattern recognition, branch point and total capillary tube length were determined following the manufacturer's guidelines (ECM625; Millipore). Image J software was used in the first instance prior to double-checking by an independent assessor. 5 random microscopic ( $\times 100)$ fields per well were included and the data are expressed as Mean \pm SD of 5 samples.

\section{Statistical analysis}

All calculations and statistical analyses were performed by using GraphPad Prism 5.0 software (San Diego, USA). T test was used to analyze the significance of any differences between two groups. The statistical significance was defined as $p<0.05$. 


\section{RESULTS}

\section{Arsenite suppressed angiogenesis of HUVECs in a dose-dependent manner}

To evaluate the effects of arsenite on the angiogenesis of HUVECs, an in vitro angiogenesis assay was performed for the cells exposed to varying concentration of arsenite for differing periods of time. Fig. 1 shows representative microscopic appearances. Cells not exposed to arsenic displayed morphologic features of angiogenesis, specifically, cells aligned themselves; there was formation of capillary tubes with or without sprouting; there was formation of closed polygons and/or complex mesh-like structures. Upon exposure to arsenic, incomplete network formation and smaller numbers of branch points or tubular structures were found. The decrease was more severe following exposure to greater concentrations of arsenite and after longer periods of time. Fig. 2 depicts the statistically analyzed results. A, B and C show the dose dependent effects on parameters of pattern recognition, branch points and total tube lengths, each reflecting a different aspect of angiogenesis. D, E and F show the IC50 values for these parameters. Clearly, arsenite inhibited angiogenesis of HUVECs in dose dependent manner. The effect was more severe when exposure time was increased from 24 to 48 hours, as reflected by a shift of the dose-effect curve for the parameters shown in A, B and C and the IC50 values shown in D, E and F. IC50 values for 24 hour arsenic exposure are $12.4 \pm 0.7 \mu \mathrm{M}$ for pattern recognition; $13.0 \pm 1.1 \mu \mathrm{M}$ for branch points and $13.5 \pm 1.7 \mu \mathrm{M}$ for total tube length, respectively. IC50 values for 48 hour arsenic exposure were $6.2 \pm 0.8 \mu \mathrm{M}$ for pattern recognition, $4.8 \pm 0.3 \mu \mathrm{M}$ for branch points and 5.0 $\pm 0.9 \mu \mathrm{M}$ for total tube length, respectively. These data indicated that arsenite suppressed the in vitro angiogenesis of HUVECs in a dose and time dependent manner. 


\section{Receptor tyrosine kinase is responsible for mediating angiogenesis of HUVECS}

It has been reported that receptor tyrosine kinase plays a crucial role in mediating angiogenesis of various malignant tumors (Jeltsch et al., 2013; Tohyama et al., 2014). Potential blocking effects of E7080 (lenvatinib), an inhibitor of multi-receptor tyrosine kinase (Wiegering et al., 2014) and CP-673451, a specific inhibitor of PDGFR-beta tyrosine kinase (Roberts et al., 2005) on angiogenesis of HUVECs were analyzed in order to identify the putative receptors mediating angiogenesis of the HUVECs and further to elucidate the possible mechanism for arsenite suppression of HUVECs angiogenesis we describe above. The results are shown in Fig. 3. Panel A shows representative microscopic appearances. The cells without exposure to any inhibitor displayed typical morphologic patterns of angiogenesis, as observed in our initial study. After treatment with CP-673451, the HUVECs showed incomplete network formation. There were also smaller numbers of branch points and tubular structures. Following treatment with E7080, there were only a few atypical morphologic patterns of angiogenesis. No branch points or tubular structures were found. B, C and D are statistically analyzed results. In the cells treated with E7080 the angiogenesis of HUVECs was almost completely blocked. The results suggest that angiogenesis of HUVECs may be mostly mediated by tyrosine kinase receptors and imply that as a putative target for the effects of arsenite on angiogenesis. In the cells treated with CP-673451, the angiogenesis of HUVECs was significantly inhibited and the three parameter respectively decreased by $20 \%$ for pattern recognition; $32 \%$ for branch point formation and $29 \%$ for total tube length. The results infer that platelet derived growth factor receptor-beta plays an important role in 
mediating angiogenesis of HUVECs, and suggest that arsenite may suppress angiogenesis of HUVECs mediated by platelet derived growth factor B chain.

\section{Arsenite suppressed the angiogenesis mediated by PDGFR-beta}

To determine whether arsenite suppression of angiogenesis was mediated by PDGFR-beta, we utilized porcine aortic endothelial cells with stably transfected human PDGF beta-receptors (PDGFR-beta/PAE cells) which have been previously employed to examine PDGF-BB triggered cytoplasmic calcium responses (Ridefelt et al., 1995). To be certain that PDGFR-beta is the only active receptor responsible for these effects, we examined the ability of CP-673451, a specific inhibitor of PDGFR-beta, to block the arsenite-induced effects. As shown in Fig. 4, cells untreated with the inhibitor (0 mM of CP-673451 in Fig. 4), displayed angiogenesis ability comparable to that of HUVECs. CP-673451 treatment completely blocked angiogenesis of the cells ( $1 \mathrm{nM}$ and $5 \mathrm{nM}$ of CP-673451 in Fig. 4). This result indicated that only PDGFR-beta is responsible for the angiogenesis of the cells. After that, the effects of arsenite were explored. As shown in Fig. 5A, the cells unexposed to arsenite presented uniform networks with dense branch points and intensive tube structures $(0 \mu \mathrm{M}$ in Fig. 5A). Compared with the unexposed cells, less typical morphologic appearances for angiogenesis were observed in the cells exposed to arsenic. In the cells exposed to higher concentration of arsenite $(40-50 \mu \mathrm{M})$ the morphologic appearances for angiogenesis completely disappeared. Figure 5B-5D are statistically analyzed results on the dose-effect of angiogenesis of the cells upon arsenic exposure. It showed clearly that arsenite suppressed the angiogenesis of the cells in a dose dependent manner. These data support the conclusion that 
arsenic suppressed the angiogenesis mediated by PDGFR-beta of the vascular endothelial cells. 


\section{DISCUSSION}

In the present study, we found that arsenite suppressed the angiogenesis of HUVECs in a dose- and time-dependent manner. The earlier study performed by Kao et al found that lower concentrations (up to $1 \mu \mathrm{M}$ ) of sodium arsenite increase vascular tube formation (Kao et al., 2003). The results of the two studies complement each other, giving the completed picture for the effects of arsenite on the angiogenesis of HUVECs.

In the present study by using E7080, an inhibitor for multi-receptor tyrosine kinase (Wiegering et al., 2014) and CP-673451, a selective inhibitor for PDGFR-beta (Roberts et al., 2005), we found that angiogenesis of HUVECs is primarily mediated by tyrosine kinase receptors and that among them, PDGFR-beta is especially important.

Platelet-derived growth factor (PDGF) is believed to be an important mitogen for connective tissue, having roles during embryonic development (Appelmann et al., 2010). It has been reported that PDGF signal pathways contribute to the development of a vascular system which involves the assembly of endothelial cells and vascular smooth muscle cells/pericytes (vSMC/PC) into many different types of blood vessels (Hellstrom et al., 1999). Their study found that the capillary endothelial cells in the endothelium of growing arteries express PDGF-B chain and that vascular smooth muscle cells/pericytes (vSMC/PC) express PDGFR-beta. PDGFBB, secreted by endothelial cells, recruits PDGFR-beta positive vSMC/PC cells and stimulates their proliferation at sites of endothelial PDGF-B expression, in the mouse (Hellstrom et al., 1999). By using porcine aortic endothelial cells with stably 
transfected human PDGFR-beta we found that arsenic suppressed the angiogenesis mediated by PDGFR-beta of vascular endothelial cells. These results indicated that a PDGF autocrine mechanism might regulate angiogenesis of human vascular endothelial cells, found in this study by application of PDGFR-beta-selective inhibitor, CP-673451, in HUVECs. Our conclusion is supported by the earlier study with bovine aortic endothelial cells showing that there are two populations of endothelial cells (angiogenic endothelial cells and nonangiogenic endothelial cells) (Battegay et al., 1994). Angiogenic endothelial cells express PDGFR-beta but not PDGF-B-chain. In contrast, non-angiogenic endothelial cells do not express PDGFR-beta, but express PDGF-B-chain. In the presence of bovine or human serum, angiogenic endothelial cells, but not non-angiogenic endothelial cells, have angiogenesis ability. This research has determined that paracrine PDGF might amplify angiogenesis via a direct action on endothelially-expressed PDGFR-beta (Battegay et al., 1994). Taken together the results indicate that arsenite suppressed angiogenesis of HUVECs in a dose-dependent manner and that angiogenesis of HUVECs was mostly mediated by tyrosine kinase receptors, among them, PDGFR-beta. 


\section{CONCLUSIONS}

In the present study, we found that arsenite suppressed angiogenesis of HUVECs in a dose-dependent manner and that angiogenesis of HUVECs was mediated by tyrosine kinase receptors, among them, PDGFR-beta. These receptors play an important role in mediating angiogenesis of HUVECs and may contribute to the injuries of blood vessel in arsenism. 


\section{ACKNOWLEDGMENTS}

This study was supported in part by the National Natural Science Foundation of China

(Grants: NSFC No. 21277057) and National Science Foundation of Jilin Province (No. 20130624003JC). We would like to express our great appreciation to Professor F. William Orr from the University of Manitoba in Canada for his help in revising the manuscript. 


\section{REFERENCES}

Appelmann, I., Liersch, R., Kessler, T., Mesters, R.M., Berdel, W.E., 2010. Angiogenesis inhibition in cancer therapy: platelet-derived growth factor (PDGF) and vascular endothelial growth factor (VEGF) and their receptors: biological functions and role in malignancy. Recent Results Cancer Res 180, 51-81.

Battegay, E.J., Rupp, J., Iruela-Arispe, L., Sage, E.H., Pech, M., 1994. PDGF-BB modulates endothelial proliferation and angiogenesis in vitro via PDGF beta-receptors. J Cell Biol 125, 917-928.

Chang, C.C., Ho, S.C., Tsai, S.S., Yang, C.Y., 2004. Ischemic heart disease mortality reduction in an arseniasis-endemic area in southwestern Taiwan after a switch in the tap-water supply system. J Toxicol Environ Health A 67, 1353-1361.

Haluska, P., Adjei, A.A., 2001. Receptor tyrosine kinase inhibitors. Curr Opin Investig Drugs 2, 280-286.

Hellstrom, M., Kalen, M., Lindahl, P., Abramsson, A., Betsholtz, C., 1999. Role of PDGF-B and PDGFR-beta in recruitment of vascular smooth muscle cells and pericytes during embryonic blood vessel formation in the mouse. Development 126, 3047-3055.

Hofer, E., Schweighofer, B., 2007. Signal transduction induced in endothelial cells by growth factor receptors involved in angiogenesis. Thromb Haemost 97, 355-363.

Jeltsch, M., Leppanen, V.M., Saharinen, P., Alitalo, K., 2013. Receptor tyrosine kinase-mediated angiogenesis. Cold Spring Harb Perspect Biol 5.

Kao, Y.H., Yu, C.L., Chang, L.W., Yu, H.S., 2003. Low concentrations of arsenic induce vascular endothelial growth factor and nitric oxide release and stimulate angiogenesis in vitro. Chem Res Toxicol 16, 460-468.

Liu, B., Feng, W., Wang, J., Li, Y., Han, X., Hu, H., Guo, H., Zhang, X., He, M., 2015. Association of urinary metals levels with type 2 diabetes risk in coke oven workers. Environ Pollut 210, 1-8.

Mannarino, E., Pirro, M., 2008. Endothelial injury and repair: a novel theory for atherosclerosis. Angiology 59, 69S-72S.

Mou, Y., Yue, Z., Wang, X., Li, W., Zhang, H., Wang, Y., Li, R., Sun, X., 2016. OCT4 Remodels the Phenotype and Promotes Angiogenesis of HUVECs by Changing the Gene Expression Profile. Int J Med Sci 13, 386-394.

Rahman, M.M., Naidu, R., Bhattacharya, P., 2009. Arsenic contamination in groundwater in the Southeast Asia region. Environ Geochem Health 31 Suppl 1, 9-21.

Ridefelt, P., Yokote, K., Claesson-Welsh, L., Siegbahn, A., 1995. PDGF-BB triggered cytoplasmic calcium responses in cells with endogenous or stably transfected PDGF beta-receptors. Growth Factors 12, 191-201.

Roberts, W.G., Whalen, P.M., Soderstrom, E., Moraski, G., Lyssikatos, J.P., Wang, H.F., Cooper, B., Baker, D.A., Savage, D., Dalvie, D., Atherton, J.A., Ralston, S., Szewc, R., Kath, J.C., Lin, J., Soderstrom, C., Tkalcevic, G., Cohen, B.D., Pollack, V., Barth, W., Hungerford, W., Ung, E., 2005. Antiangiogenic and antitumor activity of a selective PDGFR tyrosine kinase inhibitor, CP-673,451. Cancer Res 65, 957-966. 
Shi, Y., Wei, Y., Qu, S., Wang, Y., Li, Y., Li, R., 2010. Arsenic induces apoptosis of human umbilical vein endothelial cells through mitochondrial pathways. Cardiovasc Toxicol 10, 153-160.

Sun, L., McMahon, G., 2000. Inhibition of tumor angiogenesis by synthetic receptor tyrosine kinase inhibitors. Drug Discov Today 5, 344-353.

Tohyama, O., Matsui, J., Kodama, K., Hata-Sugi, N., Kimura, T., Okamoto, K., Minoshima, Y., Iwata, M., Funahashi, Y., 2014. Antitumor activity of lenvatinib (e7080): an angiogenesis inhibitor that targets multiple receptor tyrosine kinases in preclinical human thyroid cancer models. J Thyroid Res 2014, 638747.

Tseng, C.H., Chong, C.K., Tseng, C.P., Centeno, J.A., 2007. Blackfoot disease in Taiwan: its link with inorganic arsenic exposure from drinking water. Ambio 36, 82-84.

Wiegering, A., Korb, D., Thalheimer, A., Kammerer, U., Allmanritter, J., Matthes, N., Linnebacher, M., Schlegel, N., Klein, I., Ergun, S., Germer, C.T., Otto, C., 2014. E7080 (lenvatinib), a multi-targeted tyrosine kinase inhibitor, demonstrates antitumor activities against colorectal cancer xenografts. Neoplasia 16, 972-981.

Woo, S.H., Park, M.J., An, S., Lee, H.C., Jin, H.O., Lee, S.J., Gwak, H.S., Park, I.C., Hong, S.I., Rhee, C.H., 2005. Diarsenic and tetraarsenic oxide inhibit cell cycle progression and bFGFand VEGF-induced proliferation of human endothelial cells. J Cell Biochem 95, 120-130.

Wu, M.M., Kuo, T.L., Hwang, Y.H., Chen, C.J., 1989. Dose-response relation between arsenic concentration in well water and mortality from cancers and vascular diseases. Am J Epidemiol 130, 1123-1132.

Zhang, X., Simons, M., 2014. Receptor tyrosine kinases endocytosis in endothelium: biology and signaling. Arterioscler Thromb Vasc Biol 34, 1831-1837. 


\section{FIGURE LEGENDS}

Fig. 1 Effects of arsenite on the angiogenesis of HUVECs

HUVECs were exposed to different concentration of arsenite, as indicated for 24 hours. Representative microscopic fields are shown. The typical branch point and tube length measured by using Image $\mathrm{J}$ software were marked by red arrow and white hexagon, respectively.

\section{Fig. 2 Arsenite suppressed the angiogenesis of HUVECs}

HUVECs were exposed to various concentration of arsenite for different periods of time, as indicated. The angiogenesis assay, cell staining and the values quantification for the pattern recognition, branch point and total capillary tube length are described in the Methods section. Dose-dependent decreases of angiogenesis were plotted by using a nonlinear regression model (A, B and C) and the data are expressed relative to that of the control cells without exposure to arsenite. IC50 values were determined based on the fitted curves (D, E and F) and the data are expressed as the mean $\pm \mathrm{SD} . \mathrm{N}=5, * \mathrm{P}<0.05$ and $* * \mathrm{P}<0.01$ versus the control cells $($ As $=0)$. 
Fig. 3 Inhibitors of tyrosine kinase receptor suppressed the in vitro angiogenesis of

\section{HUVECs}

The angiogenesis of HUVECs was evaluated in the absence (control in A) or presence of CP-673451 (5 nM), a selective inhibitor of PDGFR or E7080 (50 nM), an active inhibitor for multiple tyrosine kinase receptors for 6 hours. The two agents only inhibit the kinase activity of the relative tyrosine kinase receptors, but do not cause a toxic effect to the cells in the concentration used in this study (Roberts et al., 2005; Wiegering et al., 2014). For quantification, the values for the pattern recognition, branch point and total capillary tube length are described in the Methods section. Representative microscopic fields are shown in Panel A. The suppressive effects of the inhibitors on angiogenesis of HUVECs are shown in B, $\mathrm{C}$ and D. The data are expressed relative to that of the control cells without exposure to the inhibitor. $\mathrm{N}=5, * \mathrm{P}<0.05$ and $* * \mathrm{P}<0.01$ versus the control cells.

Fig. 4 Selective inhibitor of PDGFR-beta CP-673451 blocked the angiogenesis of PDGFR-beta/PAE cells

The angiogenesis of PDGFR-beta/PAE cells was evaluated in the absence or presence of different concentration of PDGFR-beta specific inhibitor CP-673451, as indicated for 6 hours. The concentration of CP-673451 used in this study was much lower than that required for causing a toxic effect on the cells, and specifically inhibits only the kinase activity of PDGFR-beta (Roberts et al., 2005). The network formations were visualized by Calcein-AM staining. Representative microscopic fields are shown. 
Fig. 5 Dose-dependent suppression of the angiogenesis of PDGFR-beta/PAE cells after exposure to arsenite

PDGFR-beta/PAE cells were exposed to various concentrations of arsenite, as indicated, for 24 hours and the angiogenesis assay and the values quantification for the pattern recognition, branch point and total capillary tube length were performed as described in method section. Representative microscopic fields are shown in A. Dose-dependent decreases of angiogenesis were plotted by using a nonlinear regression model (B, C and D). The data are expressed relative to that of the control cells without exposure to arsenite. $\mathrm{N}=5, * \mathrm{P}<0.05$ and $* * \mathrm{P}<0.01$ versus the control cells $(\mathrm{As}=0)$. 

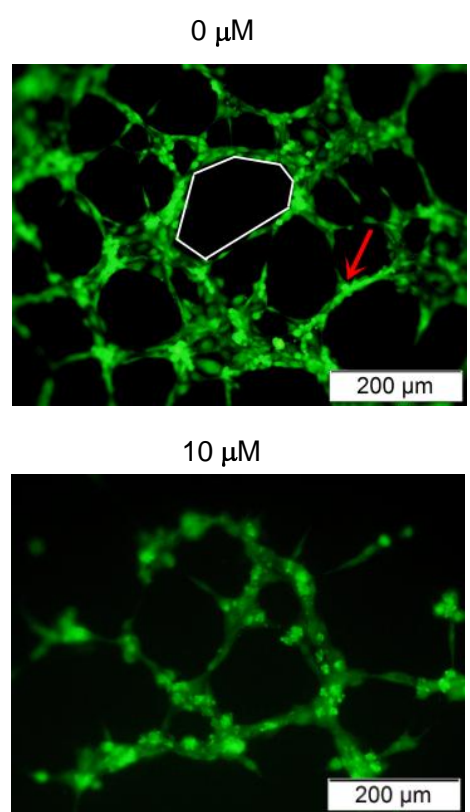

$0 \mu \mathrm{M}$
$2.5 \mu \mathrm{M}$

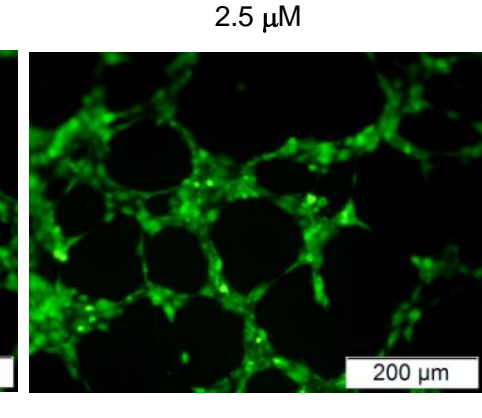

$20 \mu \mathrm{M}$

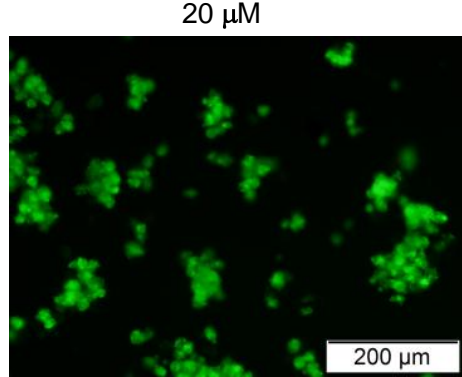

$5 \mu \mathrm{M}$

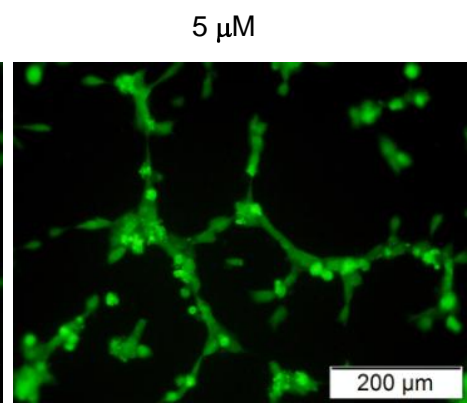

$30 \mu \mathrm{M}$

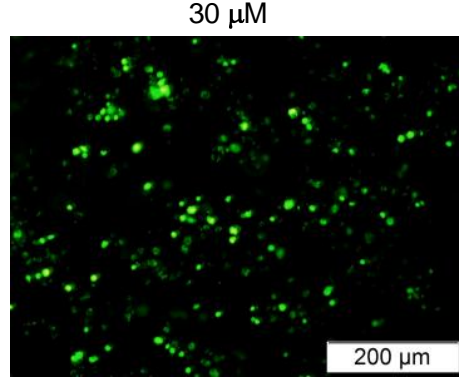

Fig. 1

\section{1}

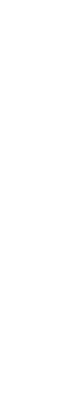

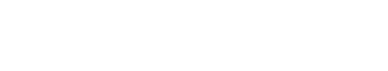

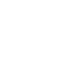$$
\text { . }
$$

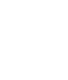



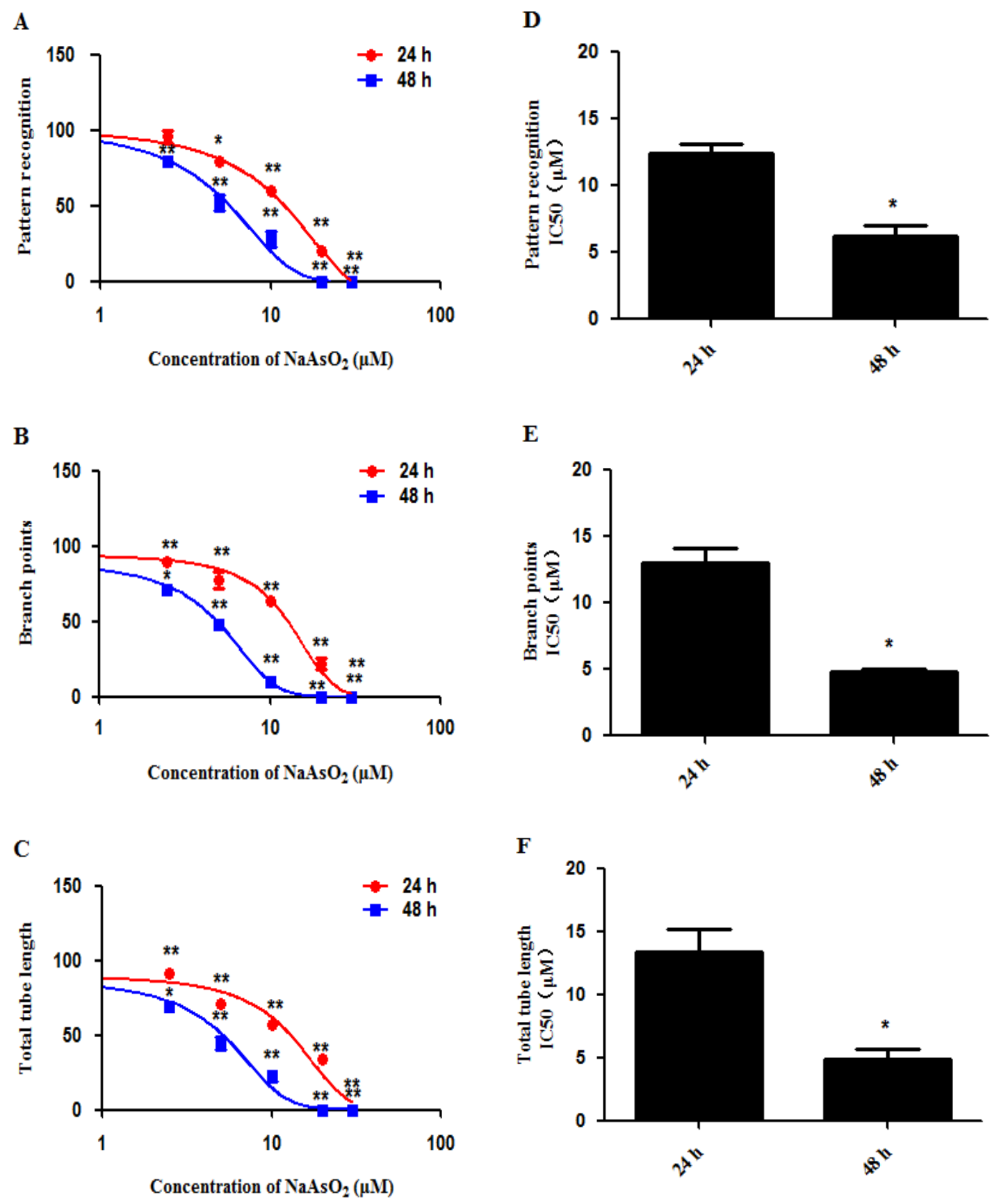

Fig. 2 
A

Control

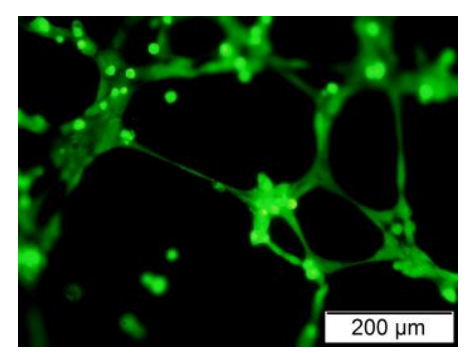

B

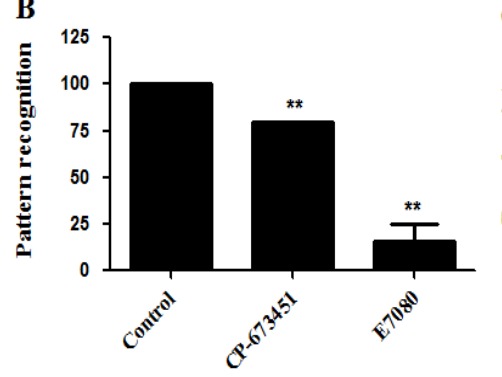

CP-673451
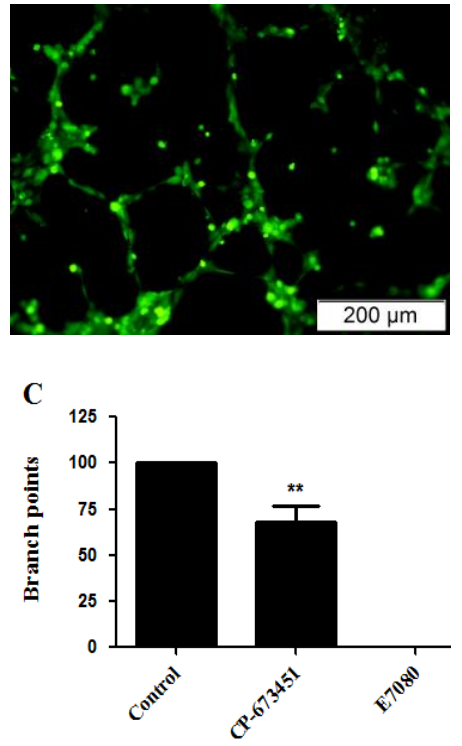

$\mathbf{E 7 0 8 0}$
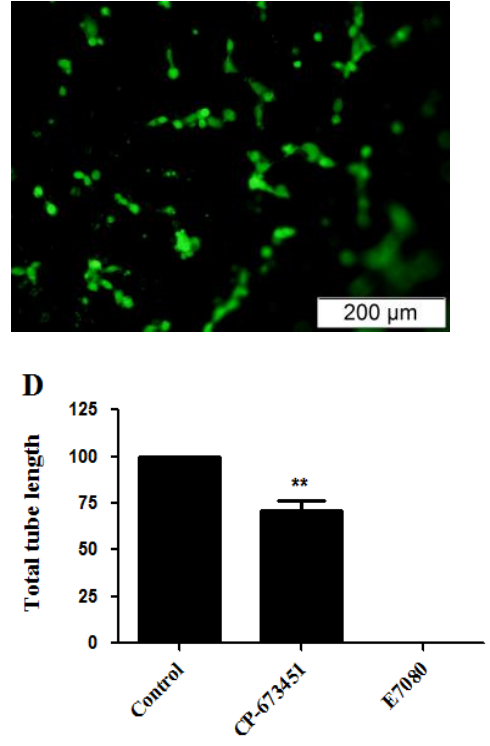

Fig. 3 

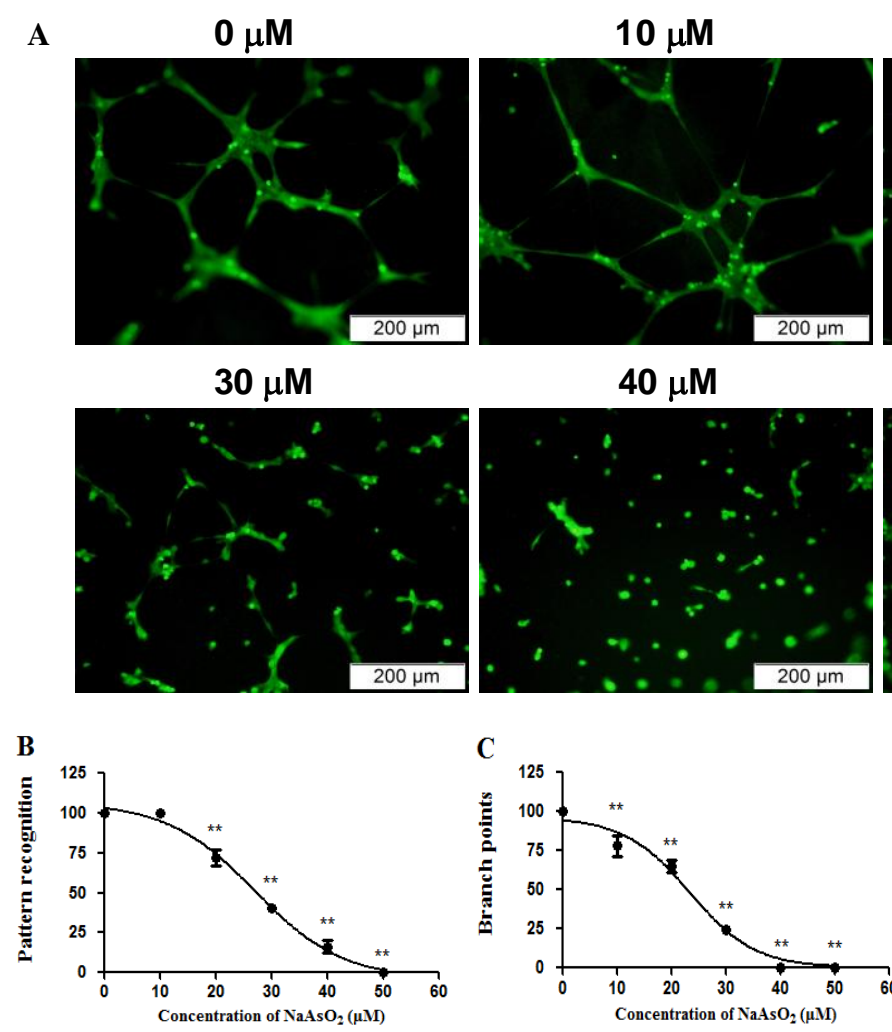

$10 \mu \mathrm{M}$

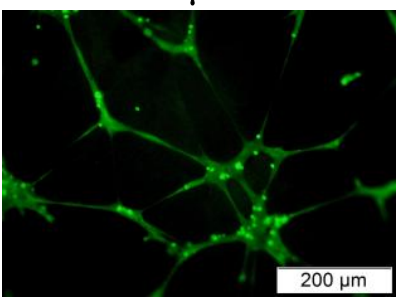

$40 \mu \mathrm{M}$

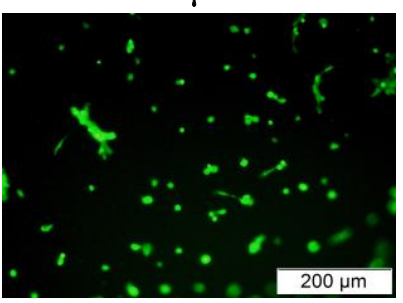

$\begin{array}{ccccc}10 & 20 & 30 & 40 & 50 \\ \text { Concentration of } \mathrm{NaAsO}_{2}(\mu \mathrm{M})\end{array}$

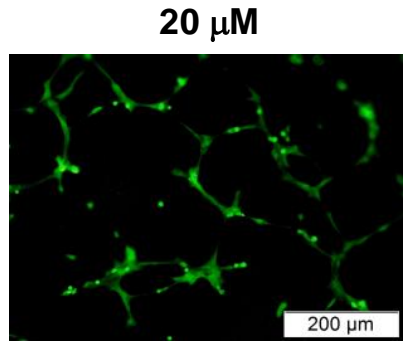

$50 \mu \mathrm{M}$
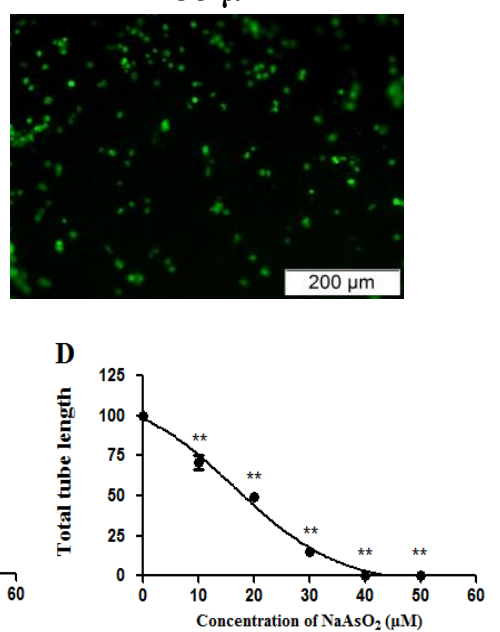

Fig. 5 


\section{An experimental arrangement for the present study}

1 To investigate the effects of sodium arsenite on the angiogenesis of human umbilical vein endothelial cells and to explore the possible mechanism involved

Sodium Arsenite

E7080 and CP-673451

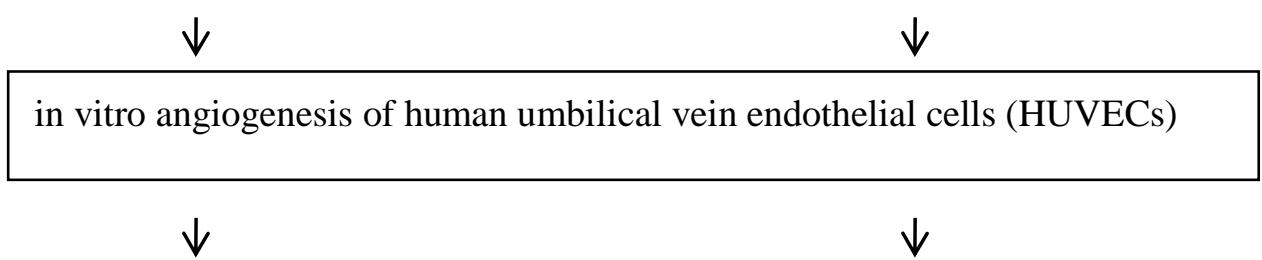

Arsenite suppressed the angiogenesis

E7080 completely prevented and

in a dose-dependent manner

CP-673451 significantly decreased

the angiogenesis

This suggested that angiogenesis of HUVECs depends on the signal pathway mediated by tyrosine kinase receptors and that among them, PDGFR-beta has an important regulatory function

2 To investigate the role of PDGFR-beta in the suppression of sodium arsenite on the angiogenesis

CP-673451

$\downarrow$

in vitro angiogenesis of porcine aortic endothelial cells which stably express human PDGFR-beta

\section{$\downarrow$}

CP-673451 completely prevented the angiogenesis
Sodium Arsenite $\left(\mathrm{NaAsO}_{2}\right)$

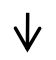

arsenite suppressed the angiogenesis in a a dose-dependent manner

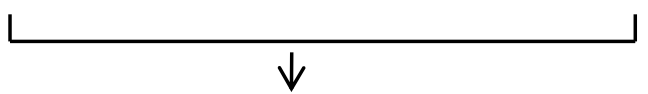

Arsenite suppressed the angiogenesis of the vascular endothelial cells mediated by PDGFR-beta and might contribute to the injuries of blood vessel in arsenism

Note:

E7080 (a global inhibitor for multiple growth factor receptors)

CP-673451 (a specific inhibitor of PDGFR-beta) 